Elsevier required licence: $\odot$ 2017. This manuscript version is made available under the CC-BYNC-ND 4.0 license http://creativecommons.org/licenses/by-nc-nd/4.0/ 


\title{
An adaptive weighted least square support vector regression for hysteresis in piezoelectric actuators
}

\author{
Xuefei Mao ${ }^{\mathrm{a}}$, Yijun Wang ${ }^{\mathrm{a}}$, Xiangdong Liu ${ }^{\mathrm{a}}$, Youguang Guo ${ }^{\mathrm{b}}$ \\ a. School of Automation, Beijing Institute of Technology, Beijing 100081, China \\ b. School of Electrical, Mechanical and Mechatronic Systems, University of Technology Sydney, Sydney, NSW \\ 2007, Australia
}

\begin{abstract}
To overcome the low positioning accuracy of piezoelectric actuators (PZAs) caused by the hysteresis nonlinearity, this paper proposes an adaptive weighted least squares support vector regression (AWLSSVR) to model the rate-dependent hysteresis of PZA. Firstly, the AWLSSVR hyperparameters are optimized by using particle swarm optimization. Then an adaptive weighting strategy is proposed to eliminate the effects of noises in the training dataset and reduce the sample size at the same time. Finally, the proposed approach is applied to predict the hysteresis of PZA. The results show that the proposed method is more accurate than other versions of least squares support vector regression for training samples with noises, and meanwhile reduces the sample size and speeds up calculation.
\end{abstract}

Keywords: piezoelectric actuator, hysteresis prediction, particle swarm optimization, adaptive weighted least squares support vector regression.

\section{Introduction}

It is known that piezoelectric actuators (PZAs) are widely used in micro-/nano-positioning systems thanks to their merits of small size, high positioning resolution, rapid response speed, and large driving force [1], [2]. Nevertheless, the major limitation of PZA is the low accuracy caused by the inherent hysteresis, which 
can produce an open-loop positioning error as much as $10-15 \%$ of the motion range [3]. To improve the positioning accuracy, a hysteresis model should be established to identify the nonlinearity of PZA.

Due to the rate-dependent property, the characteristic of the piezoelectric hysteresis is dependent not only on the amplitude but also on the frequency of input voltage signals. Hence, modeling the hysteresis using the traditional rate-independent hysteresis models such as the Preisach model [4], [5], Prandtl-Ishlinskii (PI) model [6], 7] and Krasnoselskii-Pokrovskii (KP) model [8] could yield errors subject to dynamic inputs with different frequencies. To characterize the rate-dependent hysteresis, some models were put forward, such as the improved Preisach model [9], [10], improved PI model [11], [12], and time series similarity model [13] - [15]. However, these models have a lot of parameters to be determined, which complicate the modeling process. [16] -[17] employ artificial neural network (ANN) to model the hysteresis nonlinearity, whereas it has some shortcomings such as overfitting and easily getting into the local extremum. In contrast, the support vector machine (SVM), based on statistical theory and structural risk minimization principle [18], outperforms ANN in terms of global optimization and generalization capability [19] - [21]. SVM can be applied to nonlinear regression problem by solving a convex quadratic programming (QP) problem and it shows good performance in hysteresis modeling [22], [23].

As an extension of SVM, the least squares support vector regression (LSSVR) overcomes the defect of slow training speed in SVM by solving a linear equation set rather than a quadratic optimization problem. Considering that the hyperparameters in LSSVR substantially affect the regression accuracy, some parameters selection methods were put forward, such as the analytical selection method [24], grid-searching method [25], gradient method [26], and intelligent optimization algorithms [27] - [30]. The intelligent optimization algorithms have attracted great attention due to their advantages of fast convergence and efficient global optimization. Particle swarm optimization (PSO), as one of intelligent optimization algorithms, is widely used in the field of parameters optimization because of its easy operation and excellent convergence ability [31], [32]. 
Although LSSVR accelerates the training process, employing the sum of squared error (SSE) loss function leads to the degradation of robustness, which means that the model is sensitive to noises. To overcome this drawback, some methods were proposed to filter out the outliers according to the results of LSSVR [33], [34]. However, this outlier detection method brings some errors due to the low robustness of LSSVR itself. Therefore, Suykens et al. proposed a weighted least squares support vector machine (WLSSVM) to reduce the influence of outliers by assigning smaller weights to the samples with larger training errors [35]. The key of this method is to select the appropriate weights of sample points and some other weighting strategies were proposed. For example, Wen et al. assigned smaller weights to the samples with large distance from other samples [36]. Cui et al. proposed an adaptive weighting method combined with outlier detection [37]. Behnasr et al. proposed a weight selection method based on Myriad function [38]. Xing et al. proposed a weighting method based on Cauchy distribution [39]. These methods improve the robustness of the model to some extent, whereas they have not considered that the solution of LSSVR has considerable redundancy, which means that the sample size can be reduced without degrading the performance of the regression model. In this paper, an adaptive weighting strategy is proposed to eliminate the noise interference and reduce the size of the training samples at the same time. By this means, the model based on adaptive weighted least squares support vector regression (AWLSSVR) can be both robust and sparse.

The rest of this paper is organized as follows. Section 2 provides a brief review of LSSVR and describes the hyperparameters optimization procedure based on PSO. Section 3 presents the WLSSVR method and the adaptive weighting strategy. In section 4, experimental results for modeling of hysteresis in a PZA based on AWLSSVR are presented. Conclusions are finally provided in section 5. 


\section{LSSVR and parameter optimization}

\subsection{LSSVR for hysteresis}

In order to convert the hysteresis multivalued mapping into an one-to-one mapping, the nonlinear regression model is established to predict the current output by combining the current and previous inputs and previous outputs as exogenous inputs [40]. That is

$$
y_{k}=f\left(\boldsymbol{x}_{k}\right)+\xi_{k}
$$

with

$$
\boldsymbol{x}_{k}=\left[\begin{array}{lllllll}
u_{k} & u_{k-1} & \cdots & u_{k-n} & y_{k-1} & \cdots & y_{k-m}
\end{array}\right]
$$

where $u_{k}$ and $y_{k}$ denote the input voltage and output displacement of the system at time instance $k, \xi_{k}$ is the prediction error, $f(\cdot)$ represents the nonlinear regression model, and $m$ and $n$ define the system orders. It is found that as the system orders increase, the training error gradually decreases, while the testing error does not change monotonously [23]. To make a compromise between testing error and training error, $m$ and $n$ are both set to 3 .

The LSSVR is employed to model the piezoelectric hysteresis and the model $f(\cdot)$ takes the form [41]

$$
y(\boldsymbol{x})=\boldsymbol{\omega}^{\mathrm{T}} \varphi(\boldsymbol{x})+b
$$

where a nonlinear function $\varphi(\boldsymbol{x})$ maps the input space into a high-dimensional

space. $\left\{\boldsymbol{x}_{k}, y_{k}\right\}_{k=1}^{N}$ is given as the training set, where $N$ is the sample size. $\boldsymbol{\omega}$ and $b$ are the parameters which can be determined by solving the following optimization problem

$$
\begin{aligned}
& \min _{\boldsymbol{\omega}, \xi, b} J(\boldsymbol{\omega}, \xi)=\frac{1}{2} \boldsymbol{\omega}^{\mathrm{T}} \boldsymbol{\omega}+\frac{1}{2} C \sum_{k=1}^{N} \xi_{k}^{2} \\
& \text { s.t. } y_{k}=\boldsymbol{\omega}^{\mathrm{T}} \varphi\left(\boldsymbol{x}_{k}\right)+b+\xi_{k}
\end{aligned}
$$


where $C$ represents the regularization factor which balances the training error and model complexity. The Lagrangian function of problem (4) is then expressed as

$$
L(\boldsymbol{\omega}, b, \xi, \alpha)=J(\boldsymbol{\omega}, \xi)-\sum_{k=1}^{N} \alpha_{k}\left[\boldsymbol{\omega}^{\mathrm{T}} \varphi\left(\boldsymbol{x}_{k}\right)+b+\xi_{k}-y_{k}\right]
$$

where $\alpha_{k}$ are the Lagrangian multipliers. The optimal solutions meet the following conditions

$$
\left\{\begin{array}{l}
\frac{\partial L}{\partial \boldsymbol{\omega}}=0 \rightarrow \boldsymbol{\omega}=\sum_{k=1}^{N} \alpha_{k} \varphi\left(\boldsymbol{x}_{k}\right) \\
\frac{\partial L}{\partial \xi_{k}}=0 \rightarrow \alpha_{k}=C \xi_{k} \\
\frac{\partial L}{\partial b}=0 \rightarrow \sum_{k=1}^{N} \alpha_{k}=0 \\
\frac{\partial L}{\partial \alpha_{k}}=0 \rightarrow \boldsymbol{\omega}^{\mathrm{T}} \varphi\left(\boldsymbol{x}_{k}\right)+b+\xi_{k}-y_{k}=0
\end{array}\right.
$$

Eliminating $\omega$ and $\xi$, the solutions are given by the following linear equations

$$
\left[\begin{array}{cc}
0 & \boldsymbol{e}_{N \times 1}^{\mathrm{T}} \\
\boldsymbol{e}_{N \times 1} & \boldsymbol{\Omega}+\boldsymbol{I}_{N} / C
\end{array}\right]\left[\begin{array}{l}
b \\
\boldsymbol{\alpha}
\end{array}\right]=\left[\begin{array}{l}
0 \\
\boldsymbol{Y}
\end{array}\right]
$$

where $\boldsymbol{e}_{N \times 1}=[1 ; 1 ; \cdots ; 1], \quad \boldsymbol{\alpha}=\left[\alpha_{1} ; \alpha_{2} ; \ldots ; \alpha_{N}\right], \quad \boldsymbol{Y}=\left[y_{1} ; y_{2} ; \ldots ; y_{N}\right], \boldsymbol{I}_{N}$ is an identity matrix, $\Omega_{i j}=\varphi^{\mathrm{T}}\left(\boldsymbol{x}_{i}\right) \cdot \varphi\left(\boldsymbol{x}_{j}\right)=K\left(\boldsymbol{x}_{i}, \boldsymbol{x}_{j}\right) . K$ is the kernel function and the radial basis function kernel is used in this paper

$$
K\left(\boldsymbol{x}_{i}, \boldsymbol{x}_{j}\right)=\exp \left(-\left\|\boldsymbol{x}_{i}-\boldsymbol{x}_{j}\right\|^{2} / 2 \sigma^{2}\right)
$$

where $\sigma$ is the kernel width parameter.

After obtaining $b$ and $\boldsymbol{\alpha}$ from (7), the regression model of LSSVR becomes

$$
y(\boldsymbol{x})=\sum_{k=1}^{N} \alpha_{k} K\left(\boldsymbol{x}_{k}, \boldsymbol{x}\right)+b
$$




\subsection{Hyperparameters optimization based on PSO}

The selection of hyperparameters $C$ and $\sigma$ in LSSVR is significant to obtain an accurate regression model. In this paper, PSO is adopted to optimize the hyperparameters due to its fast convergence and robustness.

PSO algorithm simulates the birds flock's behavior of preying on food and searching for the optimal position. PSO consists of a swarm of interacting particles searching in an $L$-dimensional search space of the problem's solutions ( $L$ is the size of hyperparameters). Each particle can be described by its current position and velocity. For instance, the position and velocity of particle $i$ at iteration $t$ can be expressed as $\boldsymbol{p}_{i}^{t}=\left\{p_{i 1}^{t}, p_{i 2}^{t}, \cdots p_{i L}^{t}\right\}$ and $\boldsymbol{v}_{i}^{t}=\left\{v_{i 1}^{t}, v_{i 2}^{t}, \cdots v_{i L}^{t}\right\}$. Each particle updates its speed and location by tracking individual best known position pbest $_{i}$ and swarm's best position gbest. The velocity and position of particle $i$ are updated according to the following two formulas

$$
\begin{gathered}
\boldsymbol{v}_{i}^{t+1}=\eta \boldsymbol{v}_{i}^{t}+c_{1} \times r_{1} \times\left(\boldsymbol{p b e s t}_{i}-\boldsymbol{p}_{i}^{t}\right)+c_{2} \times r_{2} \times\left(\text { gbest }-\boldsymbol{p}_{i}^{t}\right) \\
\boldsymbol{p}_{i}^{t+1}=\boldsymbol{p}_{i}^{t}+\boldsymbol{v}_{i}^{t+1}
\end{gathered}
$$

where $\eta$ denotes the inertia weight, $c_{1}$ and $c_{2}$ are the learning factors, and $r_{1}$ and $r_{2}$ are random numbers between 0 and 1 .

The performance of each particle is evaluated by the prediction error using cross-validation. That is

$$
f(C, \sigma)=\frac{1}{N_{\text {test }}} \sum_{k=1}^{N_{\text {test }}}\left(y_{k}-\hat{y}_{k}\right)^{2}
$$

where $y_{k}$ and $\hat{y}_{k}$ are the $k$ th actual output and predicting output, and $N_{\text {test }}$ is the size of test samples.

Thus, the algorithm steps of hyperparameters selection using PSO are provided as follows:

1. Establish PSO with a group of particles with random positions and velocities.

2. For each particle $i$, build the regression model and predict the outputs for test samples, and evaluate the particle's performance using (10). 
3. Replace pbest $\boldsymbol{p}_{i}$ with the particle $i$ if the latter is superior. Replace gbest with the best particle of the population if the latter is superior.

4. Update the velocity and position of each particle based on (9).

5. Repeat steps 2-4 until the stop criteria are satisfied.

\section{Adaptive WLSSVR}

In this section, an adaptive WLSSVR is proposed to mitigate the effects of noises in the training samples. In this algorithm, the weight is assigned to each sample datum according to the training error of unweighted LSSVR. The proposed weighting method can not only eliminate the noise interference but also reduce the sample size.

\subsection{WLSSVR}

WLSSVR can be obtained by weighting the error terms $\xi_{k}^{2}$ in (4). Supposing the weights are $v_{k}(k=1,2, \cdots, N)$, the optimization problem is changed as follows [35]

$$
\begin{aligned}
& \min _{\omega, \xi, b} J(\boldsymbol{\omega}, \xi)=\frac{1}{2} \boldsymbol{\omega}^{\mathrm{T}} \boldsymbol{\omega}+\frac{1}{2} C \sum_{k=1}^{N} v_{k} \xi_{k}^{2} \\
& \text { s.t. } y_{k}=\boldsymbol{\omega}^{\mathrm{T}} \varphi\left(\boldsymbol{x}_{k}\right)+b+\xi_{k}
\end{aligned}
$$

The corresponding Lagrangian function takes the same form as (5). The optimal solutions are satisfied by

$$
\left\{\begin{array}{l}
\frac{\partial L}{\partial \boldsymbol{\omega}}=0 \rightarrow \boldsymbol{\omega}=\sum_{k=1}^{N} \alpha_{k} \varphi\left(\boldsymbol{x}_{k}\right) \\
\frac{\partial L}{\partial \xi_{k}}=0 \rightarrow \alpha_{k}=C v_{k} \xi_{k} \\
\frac{\partial L}{\partial b}=0 \rightarrow \sum_{k=1}^{N} \alpha_{k}=0 \\
\frac{\partial L}{\partial \alpha_{k}}=0 \rightarrow \boldsymbol{\omega}^{\mathrm{T}} \varphi\left(\boldsymbol{x}_{k}\right)+b+\xi_{k}-y_{k}=0
\end{array}\right.
$$

$\alpha$ and $b$ are obtained by the solving the following linear equations 


$$
\left[\begin{array}{cc}
0 & \boldsymbol{e}_{1 \times N}^{\mathrm{T}} \\
\boldsymbol{e}_{1 \times N} & \boldsymbol{\Omega}+\boldsymbol{V} / C
\end{array}\right]\left[\begin{array}{l}
b \\
\boldsymbol{\alpha}
\end{array}\right]=\left[\begin{array}{c}
0 \\
\boldsymbol{Y}
\end{array}\right]
$$

with the diagonal matrix $V=\operatorname{diag}\left\{1 / v_{1}, 1 / v_{2}, \ldots 1 / v_{N}\right\}$.

Finally, the WLSSVR is expressed in the same form as (8)

\subsection{Adaptive weighting strategy}

The core idea of WLSSVR is how to determine the weights $v_{k}$ to achieve a robust and accurate regression model. For a sample datum, the bigger the training error is, the more likely it is an outlier. Motivated by this idea, smaller weights are assigned to those sample data with large errors to eliminate the disturbance of the outliers.

In addition, the optimal condition $\alpha_{k}=C v_{k} \xi_{k}$ in (12) shows that the support values $\alpha_{k}$ are directly proportional to weights $v_{k}$. Therefore, samples with relatively large training errors can be deleted in the regression model by assigning zero weights to them. This method can not only eliminate the effect of outliers on the model performance, but also prune some useless samples.

Hence, the adaptive weighting function is proposed as follows [35]

$$
v_{k}=\left\{\begin{array}{cc}
e^{-k_{1}\left(\frac{\xi_{k}}{\hat{s}}\right)^{2}} & \left|\frac{\xi_{k}}{\hat{s}}\right|<k_{2} \\
0 & \left|\frac{\xi_{k}}{\hat{s}}\right| \geq k_{2}
\end{array}\right.
$$

with

$$
\hat{s}=\frac{I Q R}{2 \times 0.6745}
$$

where $\xi_{k}$ is the training error in the $k$ th sample, $k_{1}$ and $k_{2}$ denote the parameters of weighting function. $\hat{s}$ is a robust estimate of standard deviation of $\xi_{k}$ and $I Q R$ is the difference between the $75^{\text {th }}$ percentile and the $25^{\text {th }}$ percentile. 
The relationship between $v$ and $\xi / \hat{s}$ is illustrated in Fig. 1. It is shown that the closer $\xi / \hat{s}$ is to 0 , the larger $v$ is, and if the absolute value of $\xi / \hat{s}$ is larger than the threshold $k_{2}, v$ is set to 0 .

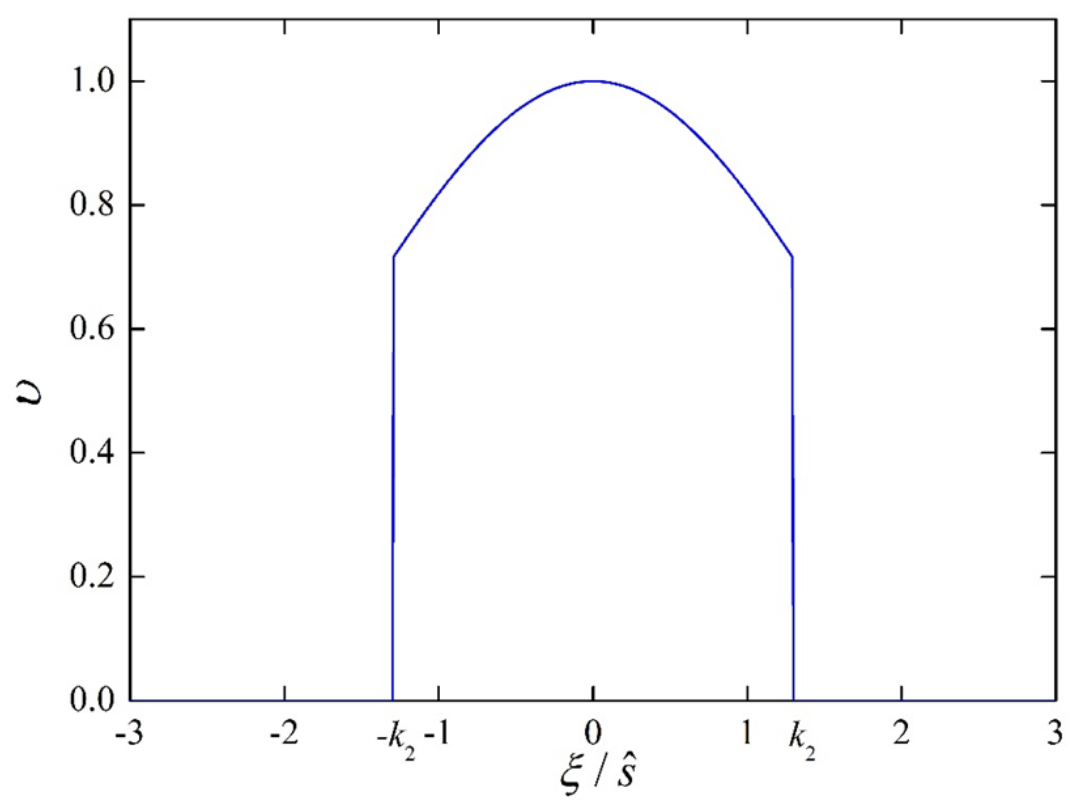

Fig. 1 Relationship between $v$ and $\xi / \hat{s}$.

\subsection{The adaptive WLSSVR algorithm}

Firstly, the optimal hyperparameters $C$ and $\sigma$ are selected using PSO. The initial fitting errors $\xi_{k}^{\prime}(k=1,2, \cdots, N)$ are obtained based upon the un-weighted LSSVR solutions. However, due to the low robustness of the LSSVR, the initial errors $\xi_{k}^{\prime}$ are not accurate enough. Thus, the iterative WLSSVR is used to obtain the error variables $\xi_{k}^{\prime}$. This method is realized using WLSSVR iteratively based on training samples and the weights are updated according to the fitting errors and weighting function until the error variables $\xi_{k}$ are converged. Finally, the weights are determined according to the weight function (14) and the errors $\xi_{k}$. The weighting function during iteration is the non-zero part of function (14): $v_{k}=\exp \left(-k_{1}\left(\xi_{k} / \hat{s}\right)^{2}\right)$. 
The adaptive WLSSVR approach is as follows:

1) Obtain the optimal hyperparameters $C$ and $\sigma$ by PSO.

2) LSSVR is applied to the model based on the training samples, and the initial fitting errors $\bar{\xi}_{k}=\bar{\alpha}_{k} / C$ are obtained.

3) Calculate the weights $\bar{v}_{k}=\exp \left(-k_{1}\left(\bar{\xi}_{k} / \hat{s}\right)^{2}\right)$.

4) Build the model by WLSSVR and calculate the fitting errors $\xi_{k}=\alpha_{k} / \bar{v}_{k} C$.

5) If the difference between $\xi_{k}$ and $\bar{\xi}_{k}$ is not small enough, let $\bar{\xi}_{k}=\xi_{k}$ and go back to step 3; or if the error variables $\xi_{k}$ are converged, determine the weights $v_{k}$ based upon (14).

6) Develop the adaptive WLSSVR model with the weights $v_{k}$.

\section{Application for modeling hysteresis in PZA}

\subsection{Experimental setup}

The experiments are carried out on a piezoelectric actuator MPT-1JRL002 (withstand-voltage range: -30 to $150 \mathrm{~V}$ and displacement resolution: $0.01 \mu \mathrm{m}$ ). Fig. 2 shows the system devices. The hardware-in-the-loop simulation system produces an analogy voltage output which is then amplified by a power amplifier to drive the PZA. The output displacement of PZA is measured by a resistance strain gauge sensor which is installed within the PZA as a micrometer and then transmitted back to the hardware-in-the-loop simulation system. Fig. 3 shows the schematic diagram of the experimental system. 


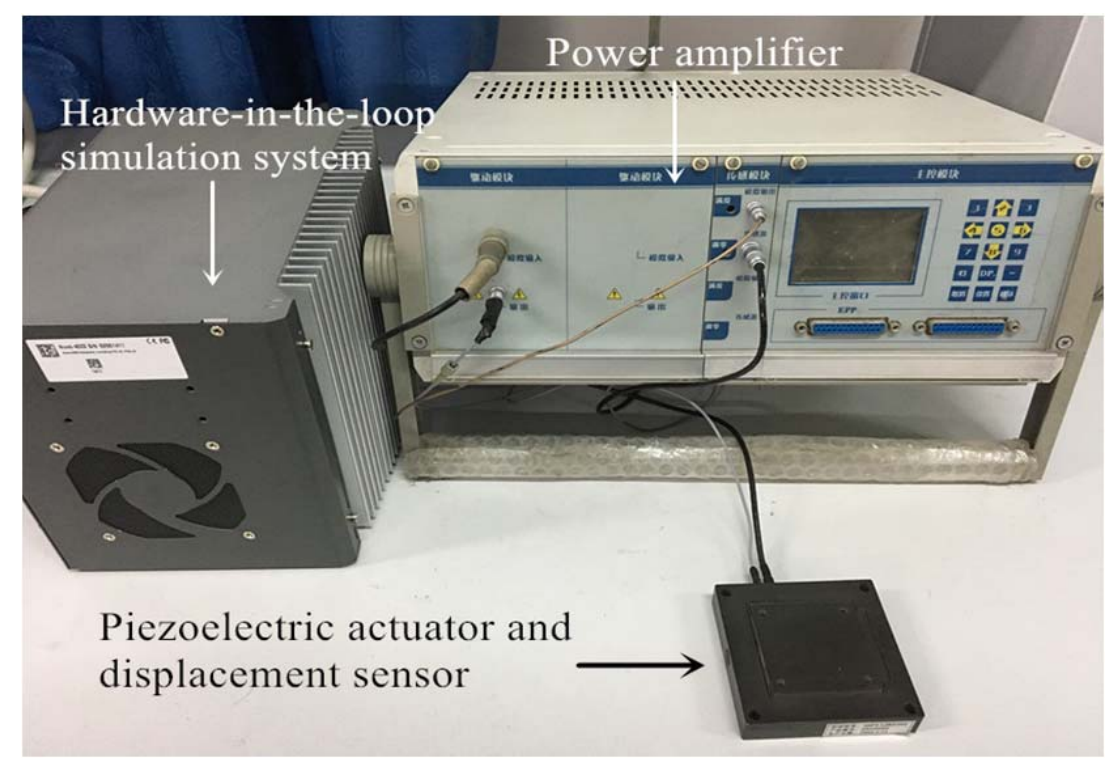

Fig. 2 Piezoelectric actuator experiment devices.

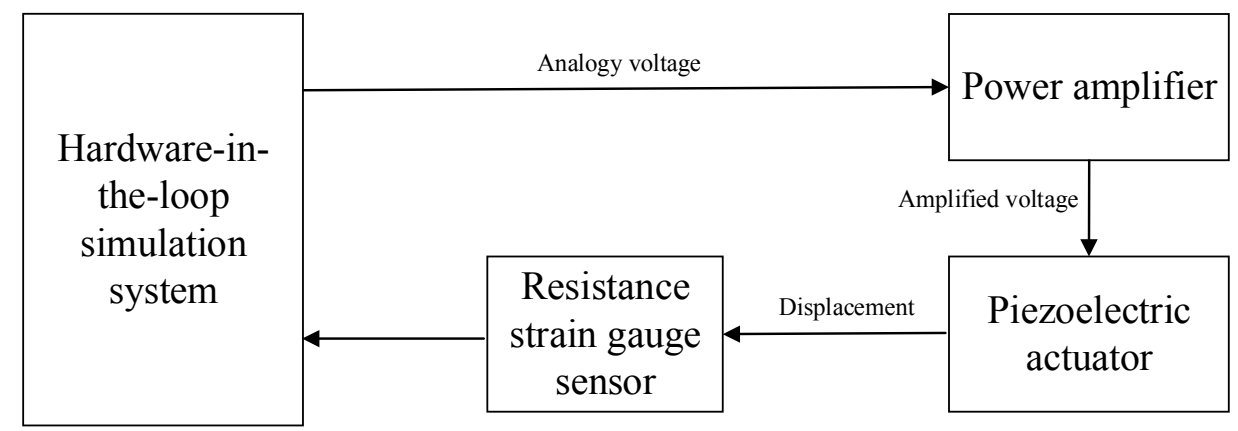

Fig. 3 Schematic diagram of piezoelectric actuator based experimental system.

\subsection{AWLSSVR model training and testing}

Considering the rate-dependent behavior of piezoelectric hysteresis, the training data must reflect the effects of input signal frequency on the response of the hysteresis. Thus, 10002 sample points shown in Fig. 4(a) is used for training, which is composed of several parts with change-rate of $2 \mathrm{~V} / \mathrm{s}, 5 \mathrm{~V} / \mathrm{s}, 10 \mathrm{~V} / \mathrm{s}, 20 \mathrm{~V} / \mathrm{s}$ and $40 \mathrm{~V} / \mathrm{s}$. The corresponding output displacement is shown in Fig. 4(b), of which 524 points randomly selected is added by Gaussian noises $(\mu=0, \sigma=0.2 \mu \mathrm{m})$ to represent the samples contaminated by outliers. 

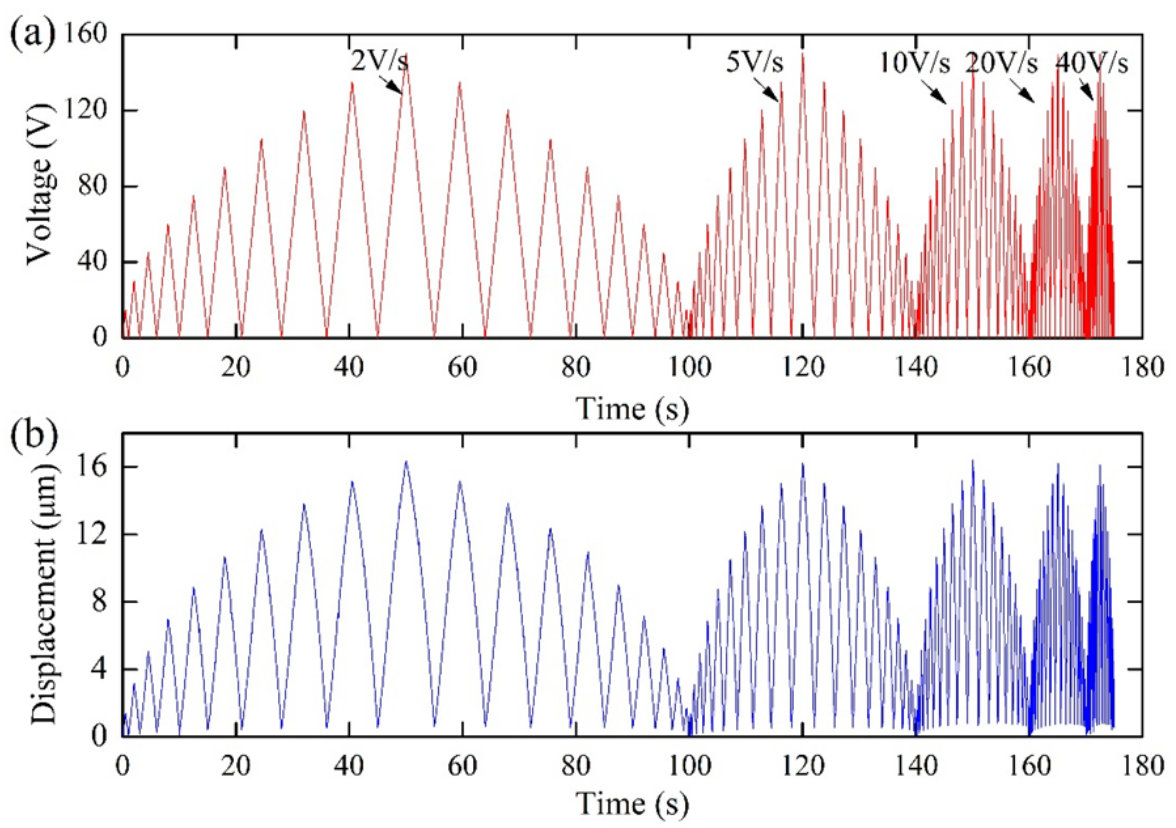

Fig. 4 The training data set. (a) The input voltage. (b) The output displacement.

Given the training data set, the hyperparameters are set as $C=8.832 \times 10^{5}$ and $\sigma$ $=0.5716$ by PSO. Then the effects of the weighting function parameters $k_{1}$ and $k_{2}$ on model performance are investigated. It is found that with the increase of $k_{1}$, the predictive accuracy of the model increases first and then decreases; as $k_{2}$ increases, the prediction capability of the model rises slowly, whereas the sparseness degrades. To make a trade-off between prediction ability and sparseness, parameters are chosen as $k_{1}=0.2$ and $k_{2}=1$.

Testing datasets with the capacity of 1378 and 1118 are generated by input voltage signals with two different waveforms as shown in Fig. 5 and each waveform corresponds to a series of test inputs with change-rate of $1 \mathrm{~V} / \mathrm{s}, 2 \mathrm{~V} / \mathrm{s}, 5 \mathrm{~V} / \mathrm{s}, 10 \mathrm{~V} / \mathrm{s}$, $20 \mathrm{~V} / \mathrm{s}, 30 \mathrm{~V} / \mathrm{s}$ and $40 \mathrm{~V} / \mathrm{s}$ respectively. The testing waveforms are chosen as random triangular waves, which are consistent with the training waveforms. 

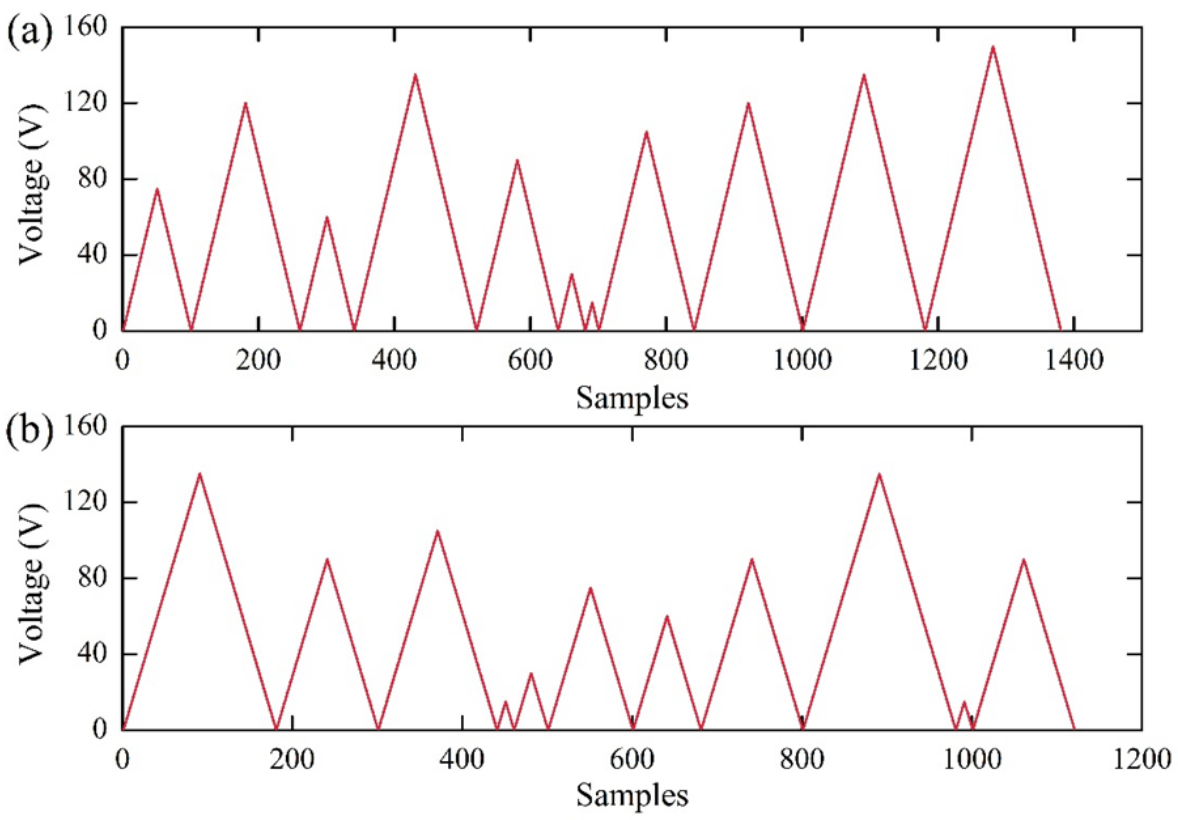

Fig. 5 The input voltage waveforms. (a) Test waveform 1. (b) Test waveform 2.

Taking the test data excited by the $10 \mathrm{~V} / \mathrm{s}$-change-rate input voltage signals as an example, the prediction results with two different waveforms are shown in Fig. 6. The output results subject to voltage signals with all the other change-rates follow the similar pattern as Fig. 6. The results show that the proposed algorithm can achieve accurate regression for the PZA hysteresis under random triangular input excitation. 

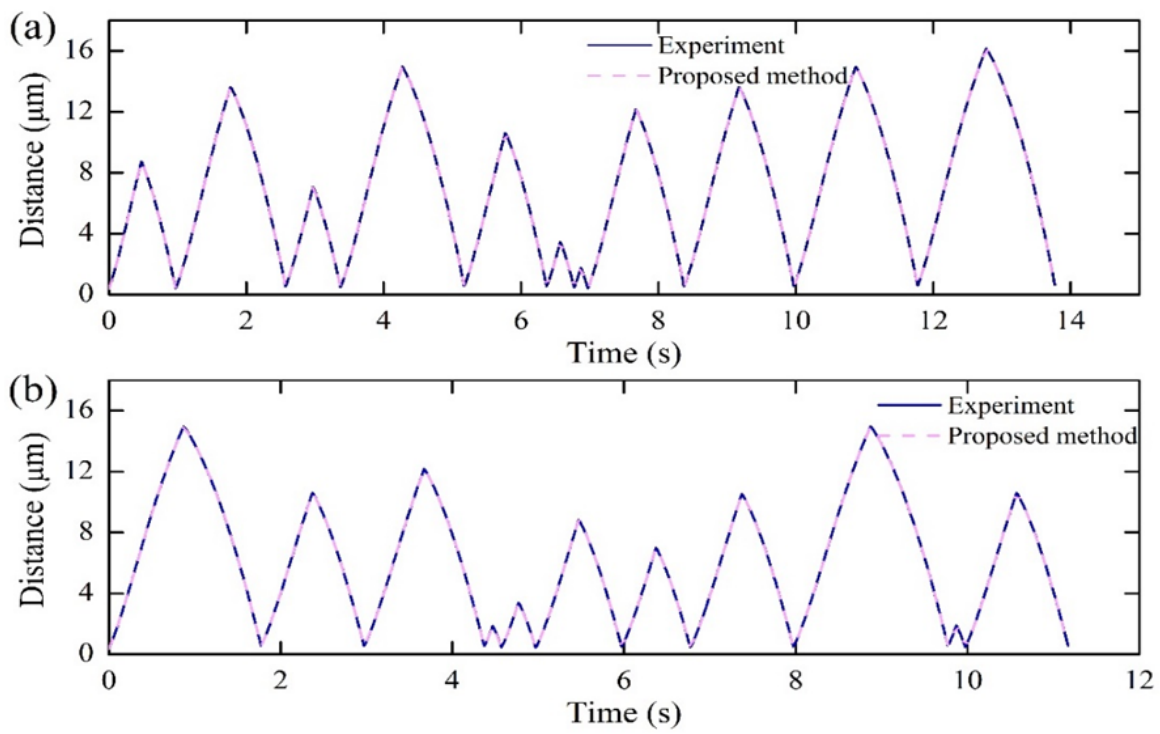

Fig. 6 Hysteresis modeling results under 10V/s-change-rate input excitation. (a) Test waveform 1. (b) Test waveform 2.

To validate the proposed model, LSSVR and Suykens' WLSSVR are also adopted to model the PZA hysteresis as comparison. The root mean squared error (RMSE) and maximal absolute error (MAXE) are employed to evaluate the robustness of regression models, which are respectively expressed as

$$
\begin{aligned}
& R M S E=\sqrt{\frac{1}{n} \sum_{k=1}^{n}\left(y_{k}-\hat{y}_{k}\right)^{2}} \\
& M A X E=\max \left(\left|y_{k}-\hat{y}_{k}\right|\right)
\end{aligned}
$$

where $y_{k}$ and $\hat{y}_{k}$ are the true value and predicted value, respectively.

The performance comparisons of three algorithms are illustrated in Fig. 7. Results indicate that when the training samples are contaminated by noises, the LSSVR produces about twice as large RMSEs as WLSSVR. Although WLSSVR is more accurate than LSSVR, it still brings 50\%-100\% larger RMSEs than the proposed algorithm. The proposed algorithm yields the best RMSEs of about 0.005 $\mu \mathrm{m}$, accounting for $0.0312 \%$ of the motion range. As for MAXE, the performance of LSSVR is comparable to that of WLSSVR and both of them produce over 4 times larger MAXEs compared with the proposed method. In contrast, MAXEs of the proposed method are within $0.1 \mu \mathrm{m}$, accounting for $0.625 \%$ of the motion range. 
Therefore, it can be concluded that the classical LSSVR is sensitive to noises, indicated by its relatively large RMSEs and MAXEs. WLSSVR produces better RMSEs than LSSVR but it can bring more than $1.9 \%$ MAXEs at some points. The reason lies in that WLSSVR relies on the training errors of LSSVR, which are less robust. On the contrary, the proposed algorithm obtains the best RMSEs and MAXEs, indicating that it is more robust than other models, regardless of dataset or evaluation criterion
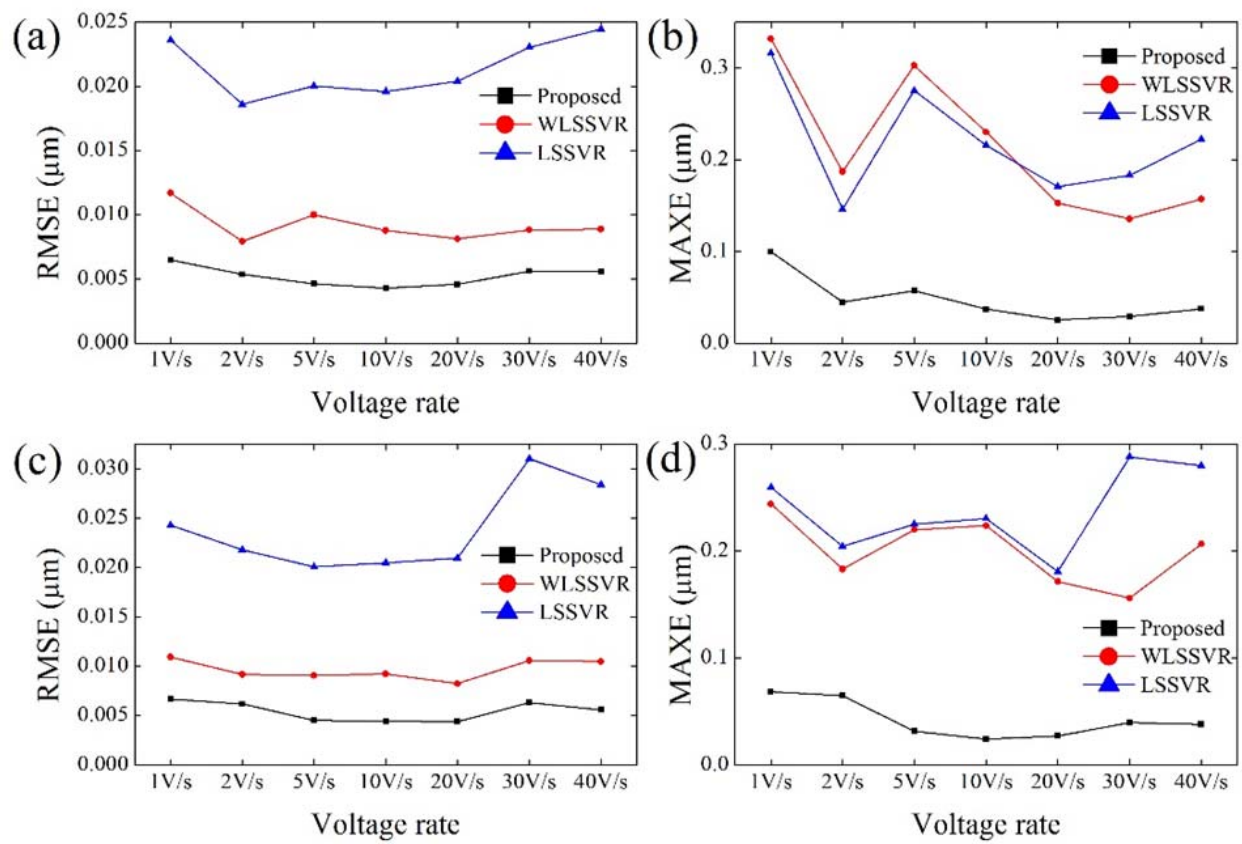

Fig. 7 Performance comparisons of LSSVR, Suykens' WLSSVR and the proposed method for hysteresis modeling. (a) and (b) RMSE and MAXE with test waveform 1. (c) and (d) RMSE and MAXE with test waveform 2.

Furthermore, the proposed algorithm reduces the sample size by $37.7 \%$ from 10,002 to 6,232 . Consequently, the average time to calculate 1,378 samples for test waveform 1 is reduced from $0.271 \mathrm{~s}$ to $0.188 \mathrm{~s}$, and the average time to calculate 1,118 samples for test waveform 2 is reduced from $0.226 \mathrm{~s}$ to $0.152 \mathrm{~s}$. Thus, the adaptive WLSSVR proposed in this paper can mitigate the effects of noises in the training samples while reducing the computational cost by $32 \%$ on average. 


\section{Conclusion}

In this paper, an adaptive WLSSVR is proposed to model the hysteresis of piezoelectric actuators when the training data contain noises. Firstly, PSO is used to select the optimal hyperparameters. Then, the weights of the samples are determined according to the adaptive weighting function and sample errors obtained by iterating WLSSVR. Test data with two different input waveforms at different change-rates are adopted to validate the robustness of the proposed method. The results show that the proposed method is more accurate than WLSSVR and unweighted LSSVR for regression with noises. Moreover, the proposed model can reduce the sample size and accelerate calculation with satisfactory regression results.

In the future work, some pruning algorithms will be investigated to further improve the sparseness of the model so that it can be applied to large-scale data.

\section{Acknowledgement}

This study was financially supported by the National Natural Science Foundation of China (Grant no. 11572035) and Beijing Natural Science Foundation of China (Grant no. 4122066).

\section{Reference}

[1] J. Schultz, J. Ueda, Nested piezoelectric cellular actuators for a biologically inspired camera positioning mechanism, IEEE Transactions on Robotics 29 (5) (2013) 1125-1138.

[2] U. Simu, S. Johansson, Analysis of quasi-static and dynamic motion mechanisms for piezoelectric miniature robots, Sensors and Actuators A: Physical 132 (2) (2006) 632-642.

[3] P. Ge, M. Jouaneh, Tracking control of a piezoceramic actuator, IEEE Transactions on Control Systems Technology 4 (3) (1996) 209-216. 
[4] I.D. Mayergoyz, Mathematical models of hysteresis, IEEE Transactions on Magnetics 22 (5) (1991) 603-608.

[5] P. Ge, M. Jouaneh, Generalized Preisach model for hysteresis nonlinearity of piezoceramic actuator, Precision Engineering 20 (2) (1997) 99-111.

[6] K. Kuhnen, Modeling, identification and compensation of complex hysteretic nonlinearities: A modified Prandtl-Ishlinskii approach, European Journal of Control 9 (4) (2003) 407-418.

[7] C.Y. Su, Q. Wang, X. Chen, S. Rakheja, Adaptive variable structure control of class of nonlinear systems with unknown Prandtl-Ishlinskii hysteresis, IEEE Transactions on Automatic Control 50 (12) (2005) 2069-2074.

[8] M.A. Krasnoselskii, A.V. Pokrovskii, System with Hysteresis, Springer, New York, 1989.

[9] R.B. Mrad, H. Hu, A model for voltage-to-displacement dynamics in piezoceramic actuator subject to dynamic-voltage excitations, IEEE/ASME Transactions on Mechatronics 7 (4) (2002) 479-489.

[10]Y. Yu, Z. Xiao, N.G. Naganathan, R.V. Dukkipati, Dynamic Preisach modeling of hysteresis for the piezoceramic actuator system, Mechanism and Machine Theory 37 (1) (2002) 75-89.

[11]W.T. Ang, P.K. Khosla, C.N. Riviere, Feedforward controller with inverse rate-dependent model for piezoelectric actuators in trajectory-tracking applications, IEEE/ASME Transactions on Mechatronics 12 (2) (2007) 134-142.

[12]M.A. Janaideh, S. Rakheja, C.-Y. Su, Experimental characterization and modeling of rate-dependent hysteresis of a piezoceramic actuator, Mechatronics 19 (5) (2009) 656-670.

[13]R. Xiong, X.-D. Liu, Z.-L. Lai, Heuristic modeling and inverse compensation of hysteresis in piezoelectric actuators based on time series similarity, Journal of Intelligent Material Systems and Structures 27 (13) (2016) 1814-1828.

[14]Z.-L. Lai, Z. Chen, X.-D. Liu, Q.-H. Wu, A novel similarity-based hysteresis empirical model for piezoceramic actuators, Sensors and Actuators A: Physical 197 (2013) 150-165.

[15]N. Dong, J. Gao, X. Liu, X. Mao, Study on the time-scale similarity of Bouc-Wen hysteresis model, in: Proceedings of 2nd International Workshop on Materials Engineering and Computer Sciences (2015) 778-781. 
[16]Xinliang Zhang, Yonghong Tan, A hybrid model for rate-dependent hysteresis in piezoelectric actuators, Sensors and Actuators A: Physical 157 (1) (2010) 54-60.

[17]R. Dong, Y. Tan, H. Chen, Y. Xie, A neural networks based model for rate-dependent hysteresis for piezoelectric actuators, Sensors and Actuators A: Physical 143 (2) (2008) 370-376.

[18] V. Vapnik, The nature of statistical learning theory, Springer, New York, 1995.

[19]K.H. Jang, T.K. Yoo, J.Y. Choi, K.C. Nam, et al., Comparison of survival predictions for rats with hemorrhagic shocks using an artificial neural network and support vector machine, in: Proceedings of 33rd Annual International Conference of the IEEE EMBS (2011) 91-94.

[20]L.J. Cao, F.H. Tay, Support vector machine with adaptive parameters in financial time series forecasting, IEEE Transactions on Neural Networks 14 (6) (2003) $1506-1518$.

[21]S. Bai, Z. Jiang, T. Wang, H. Lai, Application of online SVR on the dynamic liquid level soft sensing, in: Proceedings of 25th Chinese Control and Decision Conference (2013) 3003-3007.

[22]Y. Ma, X. Zhang, M. Xu, S. Xie, Hybrid model based on Preisach and support vector machine for novel dual-stack piezoelectric actuator, Mechanical Systems and Signal Processing 34 (1-2) (2013) 156-172.

[23]P.-K. Wong, Q. Xu, C.-M. Vong, H.-C. Wong, Rate-dependent hysteresis modeling and control of a piezostage using online support vector machine and relevance vector machine, IEEE Transactions on Industrial Electronics 59 (4) (2012) 1988-2001.

[24]V. Cherkassky, Y. Ma, Practical selection of SVM parameters and noise estimation for SVM regression, Neural Networks 17 (2004) 113-126.

[25]C.-C. Chang, C.-J. Lin, Training v-support vector classifiers: Theory and algorithms, Neural Computation 13 (2001) 2119-2147.

[26]S. Tao, D. Chen, W. Hu, Gradient algorithm for selecting hyper parameter of LSSVM in process modeling, Journal of Chemical Industry and Engineering 58 (6) (2007) 1514-1517.

[27]W. Zhao, T. Tao, E. Zio, W. Wang, A novel hybrid method of parameters tuning in support vector regression for reliability prediction: Particle swarm optimization combined with analytical selection, IEEE Transactions on Reliability 65 (3) (2016) 1393-1405. 
[28]R.G. Gorjaei, R. Songolzadeh, M. Torkaman, M. Safari, G. Zargar, A novel PSO-LSSVM model for predicting liquid rate of two phase flow through wellhead chokes, Journal of Natural Gas Science and Engineering 24 (2015) 228237.

[29] W.-C. Hong, Y. Dong, L.-Y. Chen, S.-Y. Wei, SVR with hybrid chaotic genetic algorithms for tourism demand forecasting, Applied Soft Computing 11 (2) (2011) 1881-1890.

[30]J. Li, J. Liu, J. Wang, Mid-long term load forecasting based on simulated annealing and SVM algorithm, Proceedings of the CSEE 31 (16) (2011) 63-66.

[31]J. Sun, X. Liu, A novel APSO-aided maximum likelihood identification method for Hammerstein systems, Nonlinear Dynamics 73 (1) (2013) 449-462.

[32]Y. Ding, L. Cheng, W. Pedrycz, K. Hao, Global nonlinear kernel prediction for large data set with a particle swarm-optimized interval support vector regression, IEEE Transactions on Neural Networks and Learning Systems 26 (10) (2015) $2521-2534$.

[33]W. Wen, Z. Hao, X. Yang, Robust least squares support vector machine based on recursive outlier elimination, Soft Computing 14 (11) (2010) 1241-1251.

[34]C.-C. Chuang, Z.-J. Lee, Hybrid robust support vector machines for regression with outliers, Applied Soft Computing 11 (1) (2011) 64-72.

[35]J.A.K. Suykens, J.D. Brabanter, L. Lukas, J. Vandewalle, Weighted least squares support vector machines: robustness and sparse approximation, Neurocomputing 48 (1-4) (2002) 85-105.

[36]W. Wen, Z. Hao, X. Yang, A heuristic weight-setting strategy and iteratively updating algorithm for weighted least-squares support vector regression, Neurocomputing 71 (16-18) (2008) 3096-3103.

[37]W. Cui, X. Yan, Adaptive weighted least square support vector machine regression integrated with outlier detection and its application in QSAR, Chemometrics and Intelligent Laboratory Systems 98 (2) (2009) 130-135.

[38]M. Behnasr, H. Jazayeri-Rad, Robust data-driven soft sensor based on iteratively weighted least squares support vector regression optimized by the cuckoo optimization algorithm, Journal of Natural Gas Science and Engineering 22 (2015) $35-41$.

[39]Y.-Z. Xing, X.-B. Wu, Z.-L. Xu, Weighted least square support vector machine based on Cauchy distribution, Control and Decision 24 (6) (2009) 937-940. 
[40]R. Napoli, L. Piroddi, Nonlinear active noise control with NARX models, IEEE Transactions on Audio, Speech, and Language Processing 18 (2) (2010) 286-295.

[41]J.A.K. Suykens, T. Van Gestel, J. De Brabanter, B. De Moor, J. Vandewalle, Least Squares Support Vector Machines, World Scientific, Singapore, 2002. 